\title{
Fast label-free optical diffraction tomography compatible with conventional wide-field microscopes
}

Rodrigo, José, Soto, Juan, Alieva, Tatiana

José A. Rodrigo, Juan M. Soto, Tatiana Alieva, "Fast label-free optical diffraction tomography compatible with conventional wide-field microscopes," Proc. SPIE 11060, Optical Methods for Inspection, Characterization, and Imaging of Biomaterials IV, 1106016 (21 June 2019); doi: 10.1117/12.2525840

SPIE. Event: SPIE Optical Metrology, 2019, Munich, Germany 


\title{
Fast label-free optical diffraction tomography compatible with conventional wide-field microscopes
}

\author{
José A. Rodrigo, Juan M. Soto, and Tatiana Alieva \\ Departamento de Óptica, Facultad de Ciencias Físicas, Universidad Complutense de Madrid, \\ Avda. Complutense S/N, E-28040, Mardid, Spain
}

\begin{abstract}
Partially coherent optical diffraction tomography (PC-ODT) is a labelfree quantitative 3D imaging technique based on the refractive index contrast. It provides fast non-interferometric speckle noise-free imaging compatible with conventional wide-field transmission microscopes, but suffers from two principal inconveniences. One of them is the missing cone problem, which is a common drawback for all tomographic modalities with limitedangle inspection, including interferometric coherent ODT. The second one, considered in this paper, is a nonhomogeneous contrast for different spatial frequency regions. Analyzing the microscope 3D optical transfer functions for various illuminations over the condenser aperture we have found that the Gaussian illumination shape is a proper one. Numerical simulations and experimental results support this finding. A future line in the development of post-deconvolution processing is also discussed.
\end{abstract}

Keywords: Optical diffraction tomography, Optical transfer functions, Label-free imaging

\section{INTRODUCTION}

Label-free optical diffraction tomography $(\mathrm{ODT})^{1}$ is an attractive imaging modality for studying weakly absorbing samples like microorganisms and cells. It provides a 3D refractive index (RI) distribution of the specimen from which important quantitative information such as volume, surface area, dry mass and other related characteristics can be calculated. However, the widely applied ODT techniques require specially designed holographic microscopes working with a coherent light source that sequentially illuminates the object from different sides by using a scanning module. ${ }^{2-6}$ Such coherent ODT (C-ODT) techniques recover the 3D RI by assembling numerous sections of the object spatial spectrum obtained for each illumination directions. This computationally demanding reconstruction algorithm requires hundreds of interferometric measurements that is incompatible with the analysis of rapidly changing processes. Moreover, C-ODT suffers from speckle noise and parasitic interference due to the coherent illumination.

Here we consider a partially coherent ODT (PC-ODT) technique ${ }^{7-9}$ that provides fast non-interferometric speckle noise-free imaging compatible with conventional wide-field transmission microscopes. Although the fundamentals of PC-ODT were established by N. Streibl ${ }^{10}$ more than thirty years ago, only recently its experimental implementation has been developed..$^{71-14}$ In PC-ODT, a weakly scattering sample is simultaneously illuminated by a LED source from all possible directions allowed by the condenser aperture of the wide-field microscope. The 3D RI distribution is directly recovered from a stack of through-focus intensity images by a deconvolution procedure which takes into account the system absorption (AOTF) and phase (POTF) optical transfer functions. Instead of including a mechanical refocusing system (e.g. piezo-stage) to provide the through-focus scanning, in our setup this is achieved by using an electrically focused tunable lens placed in the Fourier conjugated plane of the detector (sCMOS camera). This is particularly beneficial when high numerical aperture (NA) immersion objectives are used.

We have experimentally proven that PC-ODT provides a 3D data acquisition with a spatial resolution similar to C-ODT but with higher tomography frame rate (10 fps) compared to the current state of the art for C-ODT (2.5 fps and 0.5 fps according to Tomocube ${ }^{15}$ and Nanolive ${ }^{16}$ companies, respectively). Such fast 3D RI monitoring

Further author information: (Send correspondence to T.A.)

T.A.: E-mail: talieva@ucm.es

Optical Methods for Inspection, Characterization, and Imaging of Biomaterials IV, edited by Pietro Ferraro,

Simonetta Grilli, Monika Ritsch-Marte, Christoph K. Hitzenberger, Proc. of SPIE Vol. 11060, 1106016

(C) 2019 SPIE · CCC code: $0277-786 X / 19 / \$ 21 \cdot$ doi: $10.1117 / 12.2525840$

Proc. of SPIE Vol. 11060 1106016-1 


\section{Illumination profiles and corresponding $x z$ POTF sections}

(a) BFI with $N A_{C}=1.2$

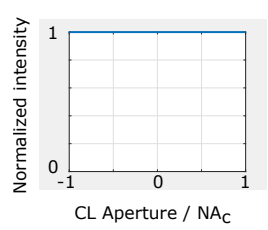

(c) Binary ring

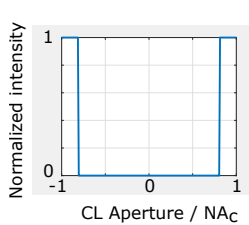

$\mathrm{CL}$ Aperture / NA (b) BFI with $\mathrm{NA}_{C}=0.5$

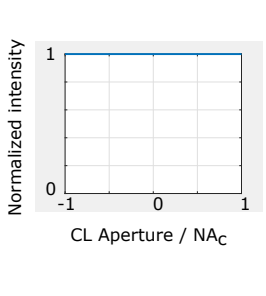

(d) IGI, $\sigma=0.5 \mathrm{NAC}$
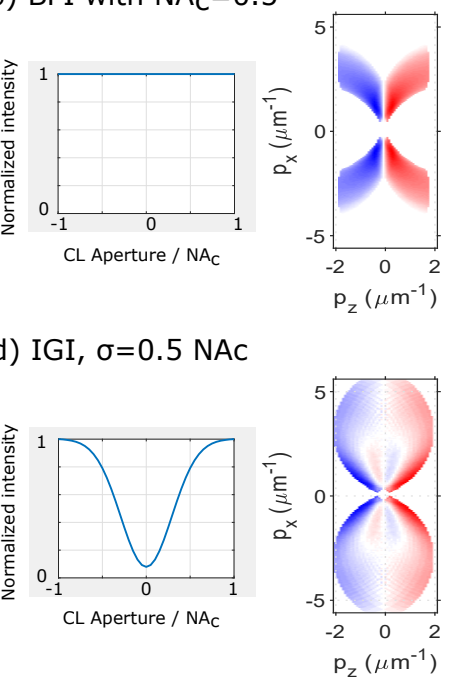

(e) GI $\sigma=0.2 \mathrm{NA}_{\mathrm{C}}+$ ring

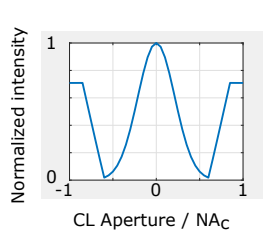

$\mathrm{CL}$ Aperture / NAC

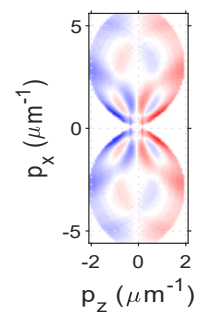

(g) GI, $\sigma=0.5 \mathrm{NA}_{C}$

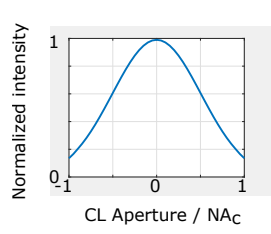

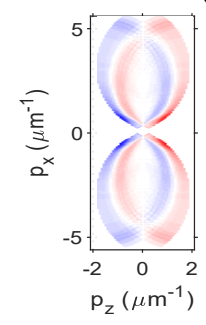

(f) $\mathrm{GI} \sigma=0.4 \mathrm{NA}_{C}+$ ring
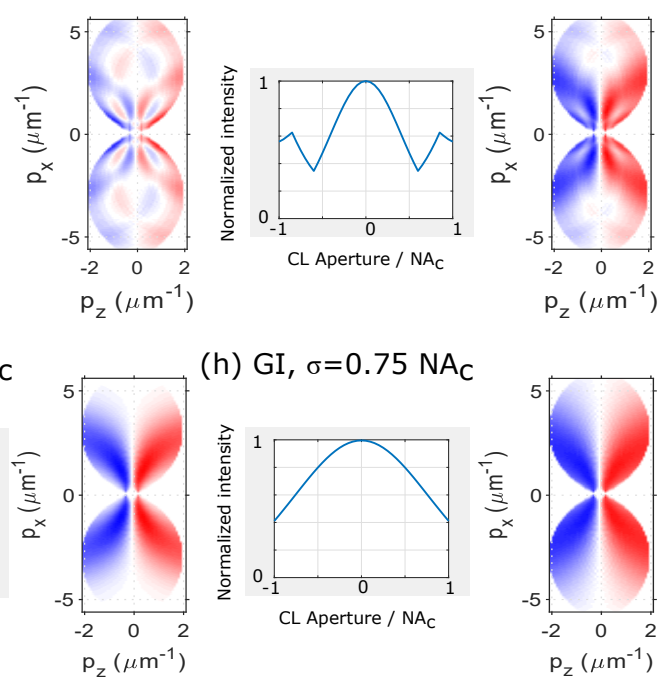

(h) GI, $\sigma=0.75 \mathrm{NA}_{\mathrm{C}}$

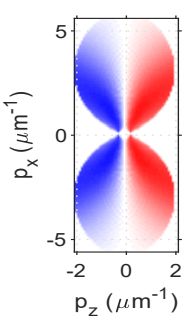

Figure 1. Sections of imaginary part of the normalized phase optical transfer functions for different illumination patterns in the condenser aperture, along with the normalized intensity profiles of such patterns. The following illuminations have been tested: (a) BFI with $\mathrm{NA}_{\mathrm{c}}=1.2$, (b) BFI with $\mathrm{NA}_{\mathrm{c}}=0.5$, (c) Binary ring, (d) IGI with $\sigma=0.5 \mathrm{NA}$, (e) Combination of GI with $\sigma=0.2 \mathrm{NA}_{\mathrm{c}}$ and binary ring, (f) Combination of GI with $\sigma=0.4 \mathrm{NA}$ and binary ring, (g) GI with $\sigma=0.5 \mathrm{NA}_{\mathrm{c}}$ and (h) GI with $\sigma=0.75 \mathrm{NA}_{\mathrm{c}}$. Except in (b), in which $\mathrm{NA}_{\mathrm{c}}=0.5$, the remaining cases have been computed with $\mathrm{NA}_{\mathrm{c}}=1.2$. All cases use $\mathrm{NA}_{\mathrm{o}}=1.4$. 
has allowed studying living cells either freely swimming or optically manipulated, for example, by using freestyle laser traps providing their all-optical confinement and transport along $3 \mathrm{D}$ trajectories. ${ }^{8}$

Nevertheless, the analysis of the POTF obtained with bright-field illumination (BFI) for the case of high NA objective and condenser lenses has revealed a low value (LV) region around the zero axial frequency that can alter the RI reconstruction apart from the well-known missing cone (MC) problem which is not considered in this work. Here we analyze different types of illumination to improve the homogeneity of the POTF and therefore the viability of the PC-ODT technique. This study verifies our previous conclusion ${ }^{9}$ that Gaussian illumination (GI) overperforms BFI in terms of POTF homogeneity. A similar study has been done to optimize the illumination pattern encoded by LED array for transport-of-intensity quantitative phase microscopy, ${ }^{17}$ however, in this case only binary encoding is possible.

\section{ILLUMINATION ENGINEERING}

There are different strategies to improve the performance of PC-ODT. One of them consists of exploiting the illumination diversity. ${ }^{12,18}$ However, it increases the number of required 3D intensity stacks (one for every illumination), the amount of time needed for their acquisition and the complexity of reconstruction algorithms. Another approach, studied here, is to design a proper illumination that allows using a single intensity stack and therefore be fast enough for 3D RI monitoring of dynamic objects. The equations involved in AOTF and POTF calculations can be found in Refs. ${ }^{7}{ }^{13}$ The considered numerical apertures of the condenser $\mathrm{NA}_{\mathrm{c}}=1.2$ and objective $\mathrm{NA}_{\mathrm{o}}=1.4$ correspond to a high resolution microscope, and the illumination wavelength is $\lambda_{0}=450 \mathrm{~nm}$. It is assumed that light at the back focal plane of the condenser is spatially incoherent, and the coherence degree of light illuminating a specimen described by the Fourier transform of its intensity distribution according to the Van Cittert-Zernike theorem. The intensity distribution of light in the sample domain is uniform. Since the ODT technique is mainly applied to low absorbing objects, we pay more attention to the POTF during the discussion.

\subsection{POTF analysis}

The 3D POTF of a perfectly aligned microscope with rotationally symmetric illumination is a pure imaginary function with rotational symmetry along the axial frequency axis $p_{z}$. This allows us consider a real function corresponding to its imaginary part further referred to as $P$. The plane $p_{z}=0$ divides the $P$-section into two symmetric halves with opposite signs, so that $P\left(p_{x}, p_{y}, p_{z}\right)=-P\left(p_{x}, p_{y},-p_{z}\right)$. Taking into account this symmetry property, only a section $P\left(p_{x}, 0, p_{z}\right)$ is analyzed in Fig. 1 , where $\left(p_{x}, p_{y}, p_{z}\right)$ denotes the spatial frequency vector. In particular, Fig. 1 displays the $P\left(p_{x}, 0, p_{z}\right)$ sections corresponding to an objective with $\mathrm{NA}_{\mathrm{o}}=1.4$ along with the corresponding illumination profiles projected on the condenser back focal plane. Among the tested illuminations included in Fig. 1 one finds BFI with two different condenser apertures $\left(\mathrm{NA}_{\mathrm{c}}=1.2\right.$ in (a) and $\mathrm{NA}_{\mathrm{c}}=0.5$ in (b)), a binary ring (c), an inverse gaussian illumination (IGI) as displayed in (d), two combinations of gaussian illumination (GI) and ring (e,f) and finally two GI with different standard deviations $(\sigma)$ of $\sigma=0.5 \mathrm{NA}_{\mathrm{c}}$ and $\sigma=0.75 \mathrm{NA}_{\mathrm{c}}$ respectively. Note that for each illumination, the $|\mathrm{POTF}|$ has been normalized to the range $[0,1]$. Regardless of the illumination pattern, there always exists a missing cone (MC) frequency region around the $p_{z}$ axis which is a well-known drawback of the wide-field microscopy that can create artifacts such as halos and longitudinal stretching in the reconstructed 3D object, ${ }^{5,19}$ in both C-ODT and PC-ODT modalities. For a same objective lens, by considering a larger $\mathrm{NA}_{\mathrm{c}}$ the $\mathrm{MC}$ is smaller and the maximum transverse resolution is higher, as observed in Fig. 1(a) and 1(b) corresponding to the BFI with $\mathrm{NA}_{\mathrm{c}}=1.2$ and $\mathrm{NA}_{\mathrm{c}}=0.5$, correspondingly. However, these advantages of high $\mathrm{NA}_{\mathrm{c}}$ are accompanied by a wider area (around the line $p_{z}=0$ ) where the absolute values of the POTF are almost negligible. We call such frequency domain as low value (LV) region. The ideal POTF has to have a smallest MC as possible and almost uniform distribution of its modulus. Therefore, the question is: Can it be achieved using an illumination different from the BFI?

Let us compare the POTFs for different illuminations. For this purpose, apart from the Fig. 1, we also show in Fig. 2 the corresponding POTF moduli $(|\mathrm{POTF}|)$ together with the histograms, which are better suited for their homogeneity analysis. The histograms of $|\mathrm{POTF}|$ for each illumination are compared in pairs with respect to the BFI case for $\mathrm{NA}_{\mathrm{c}}=1.2$. Since the high $\mathrm{NA}_{\mathrm{c}}$ is considered it is good to preserve the high resolution usually associated with the periphery illumination of the condenser aperture. Then we start our analysis by considering an annular illumination (see Fig. 1 (c) and Fig. 2 (d)). In this case we observe that the $|\mathrm{POTF}|$ distribution is 

(a) Illuminations in
condenser plane

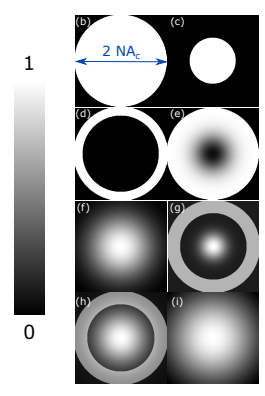

(d) Binary ring

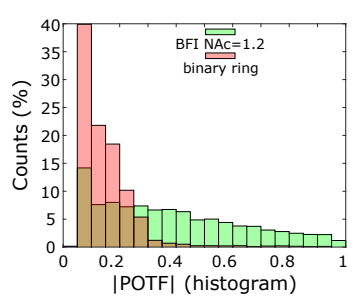

(f) $\mathrm{GI} \sigma=0.2 \mathrm{NA}_{\mathrm{C}}+$ ring

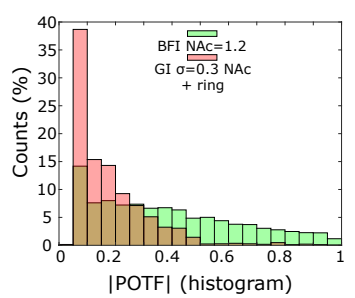

(h) GI, $\sigma=0.5 \mathrm{NA}_{\mathrm{C}}$

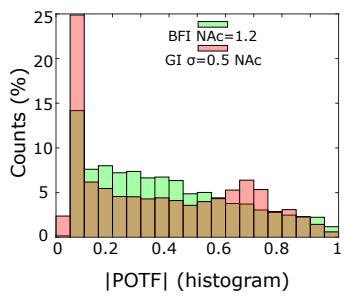

(b) Reference (BFI with $\mathrm{NA}_{C}=1.2$ )

(c) BFI with $N A_{C}=0.5$
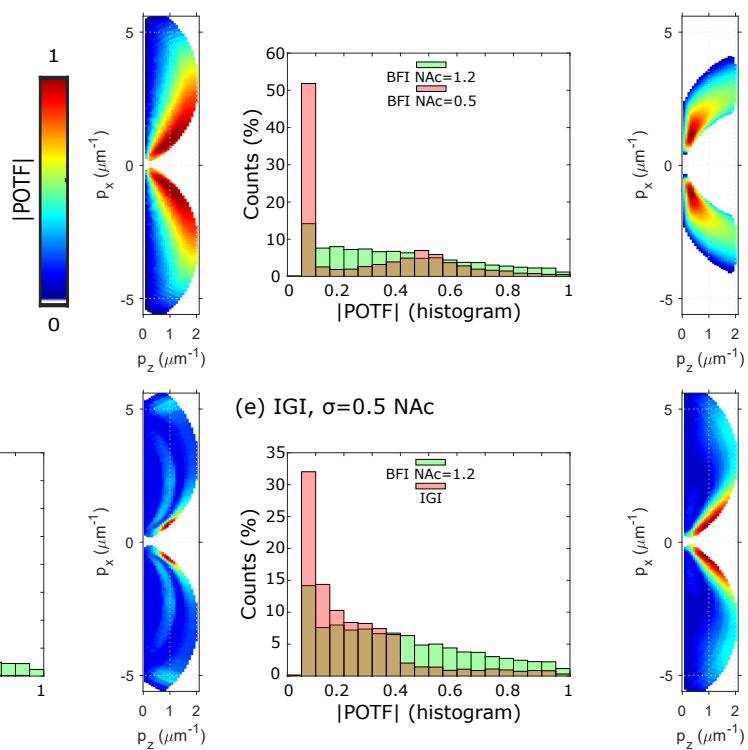

(e) IGI, $\sigma=0.5$ NAC
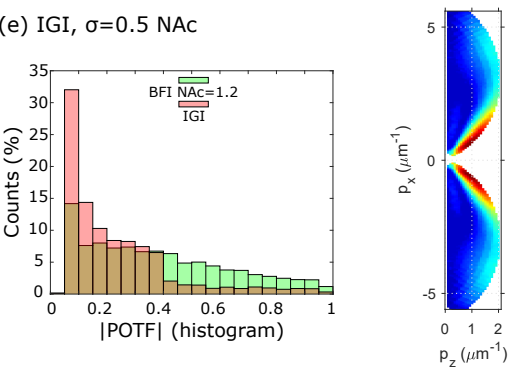

(g) GI $\sigma=0.4 \mathrm{NA}_{\mathrm{C}}+$ ring
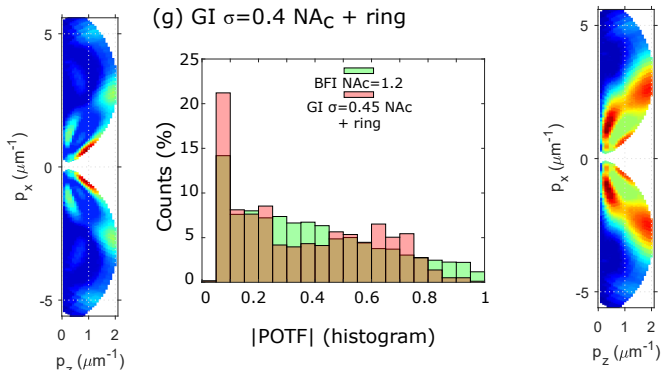

(i) GI, $\sigma=0.75 \mathrm{NA}_{\mathrm{C}}$
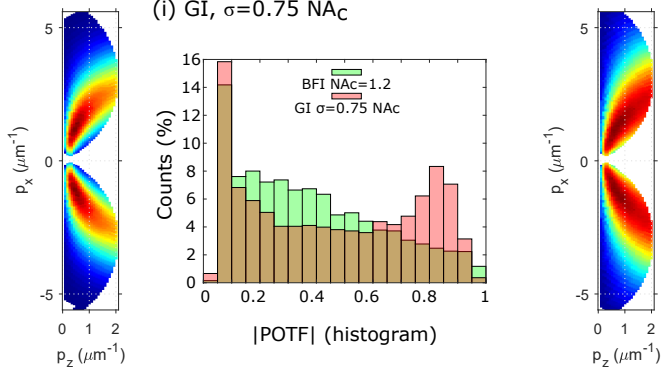

Figure 2. Normalized POTF modulus (only the right part corresponding to the section of positive axial frequencies is presented) for several illuminations, along with the $|\mathrm{POTF}|$ histogram comparing each case (rose color) with BFI $\mathrm{NA}_{\mathrm{c}}=1.2$ (green color). (a) Illumination projected in the condenser aperture. (b) BFI with $\mathrm{NA}_{\mathrm{c}}=1.2$ (reference illumination), (c) BFI with $\mathrm{NA}_{\mathrm{c}}=0.5$, (d) Binary ring, (e) IGI with $\sigma=0.5 \mathrm{NA}_{\mathrm{c}}$, (f) Combination of GI with $\sigma=0.2 \mathrm{NA}$ and binary ring, (g) Combination of GI with $\sigma=0.4 \mathrm{NA}_{\mathrm{c}}$ and binary ring, (h) GI with $\sigma=0.5 \mathrm{NA}$ and (i) GI with $\sigma=0.75 \mathrm{NA}_{\mathrm{c}}$. Except in (c), in which $N A_{c}=0.5$, the remaining cases have been computed with $N A_{c}=1.2$. All cases use $N A_{o}=1.4$. 


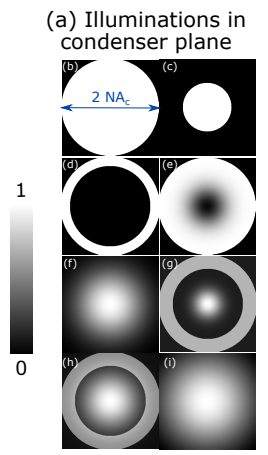

(d) Binary ring

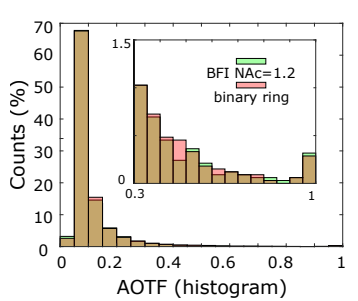

(f) GI $\sigma=0.2 \mathrm{NA}_{C}+$ ring

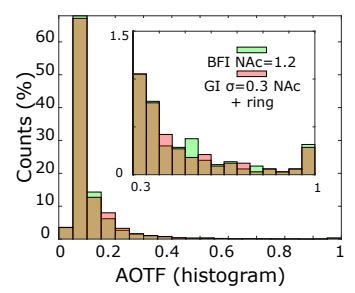

(h) GI, $\sigma=0.5 \mathrm{NA}_{\mathrm{C}}$

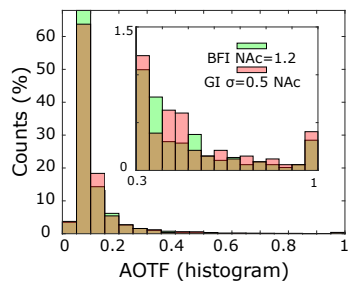

(b) Reference $\left(B F I\right.$ with $\left.N A_{C}=1.2\right)$ (c) BFI with $N A_{C}=0.5$
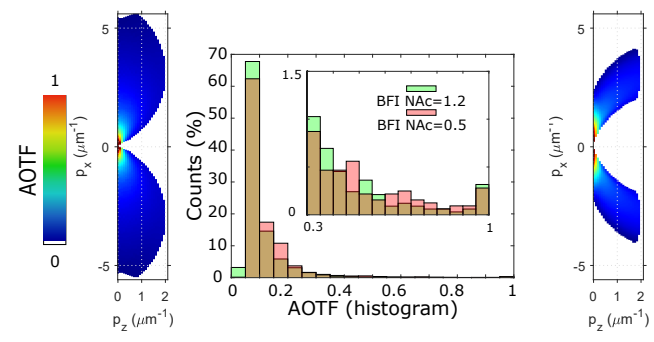

(e) IGI, $\sigma=0.5 \mathrm{NAC}$
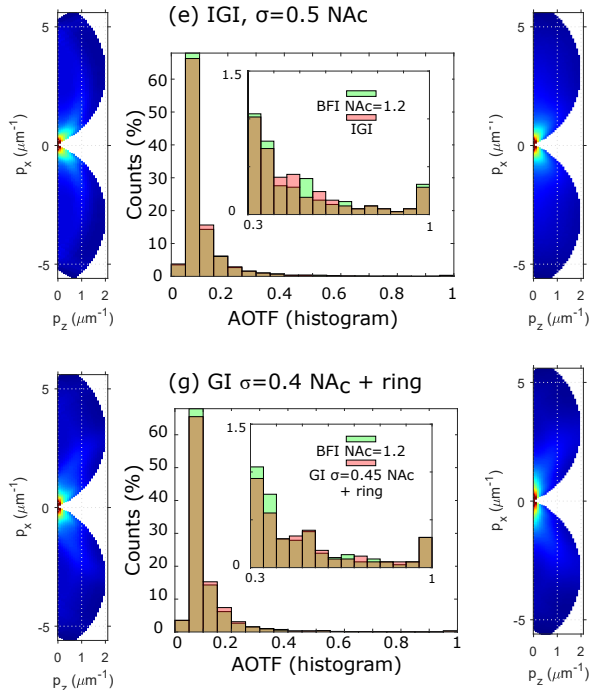

(g) GI $\sigma=0.4 \mathrm{NA}_{\mathrm{C}}+$ ring
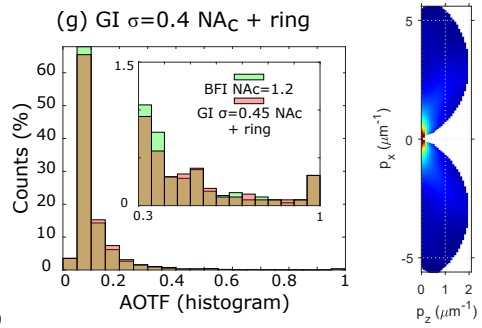

(i) GI, $\sigma=0.75 \mathrm{NA}_{\mathrm{C}}$
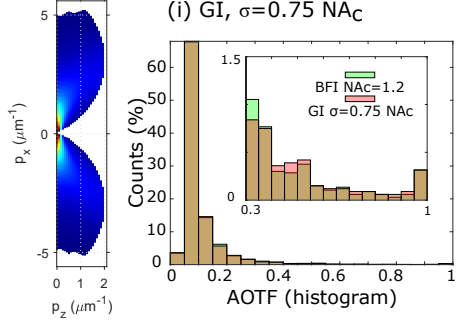

Figure 3. Normalized AOTF (only the right part corresponding to the section of positive axial frequencies is presented) for several illuminations, along with the AOTF histogram comparing each case (rose color) with BFI NA $\mathrm{c}_{\mathrm{c}}=1.2$ (green color). (a) Illumination projected in the condenser aperture. (b) BFI with $\mathrm{NA}_{\mathrm{c}}=1.2$ (reference illumination), (c) BFI with $\mathrm{NA}_{\mathrm{c}}=0.5$, (d) Binary ring, (e) IGI with $\sigma=0.5 \mathrm{NA}_{\mathrm{c}}$, (f) Combination of GI with $\sigma=0.2 \mathrm{NA}_{\mathrm{c}}$ and binary ring, (g) Combination of GI with $\sigma=0.4 \mathrm{NA}_{\mathrm{c}}$ and binary ring, (h) GI with $\sigma=0.5 \mathrm{NA}_{\mathrm{c}}$ and (i) GI with $\sigma=0.75 \mathrm{NA}_{\mathrm{c}}$. Except in (c), in which $N A_{c}=0.5$, the remaining cases have been computed with $\mathrm{NA}_{\mathrm{c}}=1.2$. All cases use $\mathrm{NA}_{\mathrm{o}}=1.4$. 
even less uniform than in the reference BFI. Moreover, as it can be seen in Fig. 1 (c) there exist sign changes into the same half of the $P$-section. This effect is undesired because the result of sign mismatching between the theoretical and experimental (realistic) ring POTFs could provide severe artifacts in RI reconstruction.

Let us now find whether or not the smoothing of the illumination ring boundary helps to yield a more uniform POTF. For this reason, we now consider an IGI defined as $I\left(\mathbf{r}_{\mathrm{c}}\right)=I_{0}-\left(I_{0} \exp \left(-r_{\mathrm{c}}^{2} / 2 \sigma^{2}\right) \operatorname{circ}\left(r_{\mathrm{c}} / \mathrm{NA}_{\mathrm{c}}\right)\right)$, where $\mathbf{r}_{\mathrm{c}}=\left(r_{x}, r_{y}\right)$ is a dimensionless coordinate vector, $I_{0}$ is the intensity maximum value and $\sigma$ denotes the standard deviation (see Fig. 1 (d) and Fig. 2 (e)). We have found a slight improvement in histogram values with respect to the ring illumination (the peak of $|\mathrm{POTF}|$ low values around 0.1 have decreased from a $40 \%$ in the ring illumination to a $30 \%$ in IGI). However, this is not significantly better than the BFI. Then, our next guess is to combine the ring illumination with a low $\mathrm{NA}_{\mathrm{c}} \mathrm{BFI}$, since as we mentioned before the such BFI exhibits a lower LV region for the low and middle transverse frequency range. This case corresponds to the Fig. 1 (e,f) and Fig. 2 $(f, g)$ where two different combinations of GI and ring-illumination (with a common center) are shown. Here the Gaussian illumination is given by $I\left(\mathbf{r}_{\mathrm{c}}\right)=I_{0} \exp \left(-r_{\mathrm{c}}^{2} / 2 \sigma^{2}\right) \operatorname{circ}\left(r_{\mathrm{c}} / \mathrm{NA}_{\mathrm{c}}\right)$. We observe that the homogeneity of the POTF modulus has not been improved with regard to the BFI. Moreover, there is a complex structure of the POTF sign changes (especially appreciable in Fig. 1 (e)) accompanied by zero-value curves (corresponding to surfaces of the full 3D POTF). From the analysis of all the aforementioned illuminations we decide to impose an additional constraint for the illumination: same POTF sign for positive (negative) axial frequencies of each $P$-section half.

Let us return to the first considered illuminations, $\mathrm{BFI}$ with $\mathrm{NA}_{\mathrm{c}}=1.2$ and $\mathrm{NA}_{\mathrm{c}}=0.5$, respectively. The first one exhibits a low MC but pronounced LV regions whereas the second one shows large MC but small LV area. Therefore, a combination of both BFI and then smoothing results in the proposed GI. Note that no sign changes exist into the same half of $P$-section with this illumination. Two cases for GI with different width (given by their standard deviation $\sigma$ ) are displayed in Fig. 1 (g,h) and Fig. 2 (h,i). We observe that GI with the higher width, $\sigma=0.75 \mathrm{NA}_{\mathrm{c}}$, provides a relevant improvement in $|\mathrm{POTF}|$ homogeneity and a reduction of the $\mathrm{LV}$ area while preserving the small $\mathrm{MC}$ region corresponding to the $\mathrm{NA}_{\mathrm{c}}=1.2$. The prize for this is further attenuation of the very high frequency region, which, in any case could not be properly resolved with the BFI due to low contrast. This study is consistent with microscopy practice. Indeed, the common recommendations in the microscope manuals is to work with the $\mathrm{NA}_{\mathrm{c}}$ closed up to $0.75 \mathrm{NA}_{\mathrm{c}}$. We also recall that such GI provides a gaussian shaping of the degree of spatial coherence in the sample domain instead of a besinc one corresponding to the BFI. Note that the secondary lobes of the besinc function may cause the appearance of undesired interference fringes of light scattered by the object that makes more questionable the application of first Born approximation in the BFI case.

\subsection{AOTF analysis}

While in the most ODT applications weakly absorbing samples are considered, absorption is often also taken into account. Thus in Refs. ${ }^{7-9}$ an effective OTF, which is a linear combination of both POTF and a weighted AOTF, is used for the RI reconstruction. Let us study the AOTF forms for the same illuminations considered in the previous section, as summarized in Fig. 3. We recall that the AOTF of each illumination has been normalized to its maximum value. The AOTF of a system presenting rotational symmetry is positive and symmetric about the plane $p_{z}=0$, that allows us considering only the AOTF section: $A\left(p_{x}, 0, p_{z}\right)$ for non-negative axial frequencies. In contrast to POTF, the maximum AOTF values are concentrated around the axis $p_{z}=0$. We observe in Fig. 3 that, in general, the AOTF is less sensitive to the illumination modification unlike the POTF. Most of normalized AOTF values are below 0.3 as can be seen from the histograms presented in Fig. 3. The largest AOTF values are concentrated in the low frequency region. This region is more elongated along the $p_{z}=0$ for BFI with $\mathrm{NA}_{\mathrm{c}}=0.5$ (Fig. 3 (c)) and GI (see Fig. 3 (h,i)) in comparison with BFI (NA $=1.2$ ), see Fig. 3 (b). On the other side, the ring-like illuminations tend to yield a more symmetric filling of the low frequency region as shown in Fig. 3 (d-g). As we can learn from the histogram in Fig. 3 (i) the GI with $\sigma=0.75 \mathrm{NA}_{\mathrm{c}}$ does not change significantly the AOTF values compared with the BFI, but it reaches a better POTF. Therefore, as POTF has a prevailing effect when weaking absorbing objects are considered, this supports our proposal of applying this Gaussian illumination for the PC-ODT instead of conventional BFI. 
(a) Phantom RI $(z=4 \mu \mathrm{m})$

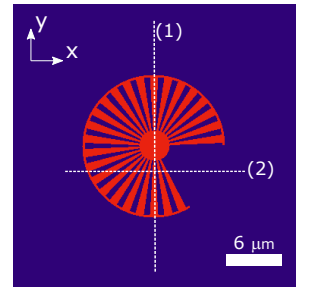

(c) Reconstructed RI ( $x z$ view) (b) Reconstructed RI (yz view)

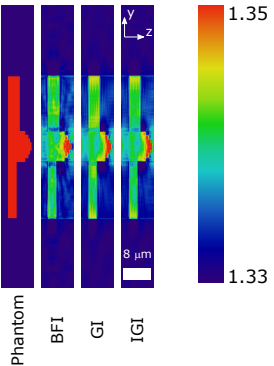

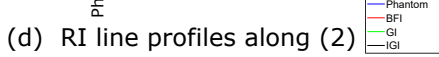
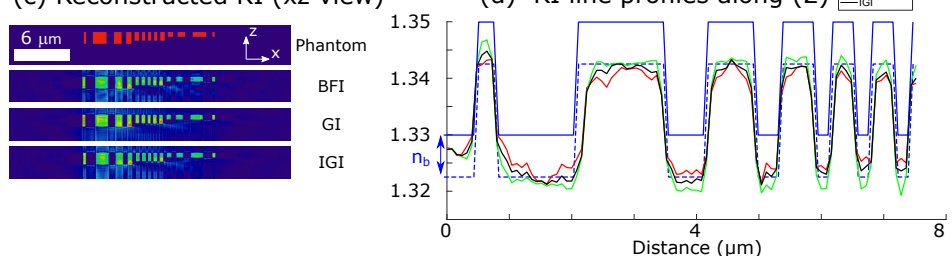

(e) Reconstructed RI ( $z=4 \mu \mathrm{m})$ with different illuminations
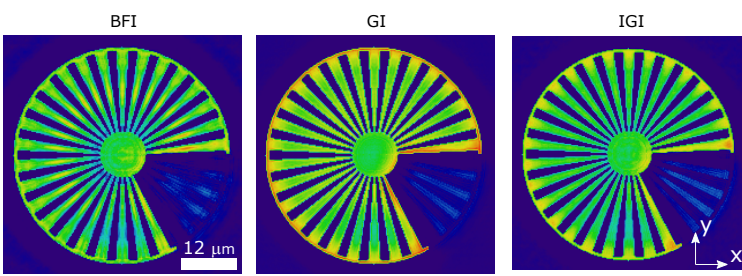

(f) Two different points of view of the 3D RI phantom

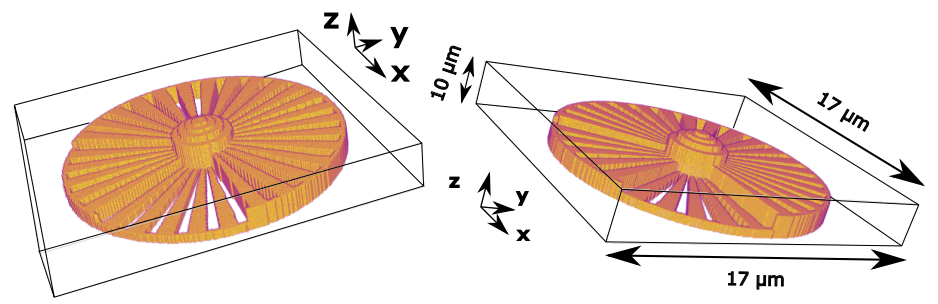

(g) 3D reconstructed RI with GI in the same points of view from (f)

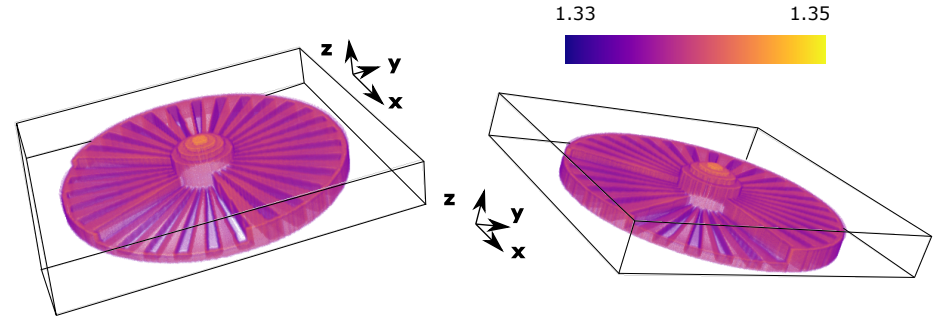

Figure 4. RI reconstruction for a simulated 3D Siemens star phantom. (a) $x-y$ slice of the phantom design. (b) $y-z$ sections corresponding to the line (1) shown in (a) for the reconstructions with BFI, GI and IGI. (c) $x-z$ sections corresponding to the line (2) shown in (a) for the reconstructed RI with BFI, GI and IGI. (d) RI profiles corresponding to half of the line (2) shown in (a). (e) Reconstructed $x-y$ RI slices corresponding to the phantom slice (a) for BFI, GI and IGI. (f) 3D visualization of the phantom. (e) 3D volume of the reconstructed RI for GI. 


\section{NUMERICAL SIMULATIONS}

In order to verify the improvements introduced by the application of the Gaussian illumination we numerically simulate the deconvolution process for three types of illumination comprising BFI $\left(\mathrm{NA}_{\mathrm{c}}=1.2\right), \mathrm{GI}\left(\sigma=0.75 \mathrm{NA}_{\mathrm{c}}\right)$ and IGI $\left(\sigma=0.5 \mathrm{NA}_{\mathrm{c}}\right)$. For this purpose we have designed a test phantom considered as a phase-only object. It consists of a structure based in a Siemens star and organized as a spiral staircase (of 3 different steps in $z$ direction) while the central area is a solid cylindrical pillar crowned with 4 concentric cylinders of decreasing radii. The RI of this structure is homogeneous $\left(n_{p}=1.35\right)$ and it is immersed in a surrounding medium mimicking the water (with a RI of $n_{m}=1.33$ ). A $2 \mathrm{D} x-y$ slice of the phantom is displayed in Fig. 4 (a), which also indicates by two lines (labeled as 1 and 2, respectively) the position of the analyzed axial sections. The RI axial sections $y-z$ corresponding to line 1 for the three simulated illuminations are displayed in Fig. 4 (b). The RI axial sections $x-z$ corresponding to line 2 are shown in Fig. 4 (c). While, Fig. 4 (d) presents the RI profile along the line 2 for each considered illumination. Then, Fig. 4 (e) displays the $x-y$ section of the reconstructed RI obtained with each illumination. By analyzing the RI sections shown in Fig. 4 (b,c,e)) we observe that GI provides better results. Indeed, the RI distribution obtained for GI is more uniform than that of the other illuminations. Furthermore, the poorer contrast of the high frequency structure (specially the region around the central pillar) obtained by BFI and IGI (see Fig. 4 (e)) is another evidence that validates that GI is the best of the tested illuminations.

The 3D visualization (created by using a volume rendering software, Tomviz) of the reconstructed RI distribution for GI displayed in Fig. 4 (g) demonstrates good agreement with the phantom structure presented in Fig. 4 (f). Nevertheless, the absolute values of the reconstructed RI significantly vary with respect to the $n_{p}$, for all the types of simulated illuminations. Particularly, as we see in Fig. 4 (d), there is a negative bias $\left(n_{b}\right)$ around the reconstructed sample. This discordance is usually associated in the literature with MC problem (see, for example, Ref. ${ }^{3}$ ). A standard post-deconvolution processing to solve this problem includes the application of iterative algorithms ${ }^{20,21}$ with certain constraints such as positivity (all values below $n_{m}$ are discarded and set to $\left.n_{m}\right)$. Here, we do not apply any iterative procedures that allows us investigating alternative ways for $\mathrm{RI}$ corrections. We observe that even if the absolute value of the reconstructed RI $\left(n_{s}\right)$ is incorrect (mainly underestimated), the difference between $n_{s}$ and the RI values in its proximity is correct $(\approx 0.02)$. Note also that the bias is varying over the sample and its modulus decreases towards the object periphery. However, the difference $n_{p}-n_{s}$ remains almost constant $(\approx 0.02)$ along the profile analyzed in Fig. $4(\mathrm{~d})$ and, generally, in the whole structure. These findings can be used in future for the development of more sophisticated algorithm for post-deconvolution processing.

\section{EXPERIMENTAL RESULTS}

Let us now consider the application of GI in the experimental PC-ODT setup reported in Refs. ${ }^{7-9}$ for the analysis of buccal mucosa squamous cells attached to a epithelial layer and immersed in water. The setup comprises a quasi-monochromatic LED illumination source $\left(\lambda_{0}=450 \mathrm{~nm}\right)$ together with a high-NA objective lens $\left(\mathrm{NA}_{\mathrm{o}}=1.4\right.$, Olympus UPLSAPO $100 \mathrm{x}$, oil immersion $\left.n_{i m m}=1.518\right)$ and a condenser lens $\left(\mathrm{NA}_{\mathrm{c}}=0.95\right)$. The transverse $\Delta x, \Delta y$ and axial $\Delta z$ Nyquist distances ${ }^{23}$ are often used for the estimation of the spatial resolution limits of ODT (see for example $\left.{ }^{24}\right)$. In our case $\Delta x=\Delta y=\lambda_{0} / 2\left(\mathrm{NA}_{\mathrm{o}}+\mathrm{NA}_{\mathrm{c}}\right) \approx 100 \mathrm{~nm}$ and $\Delta z=\lambda_{0} / 2\left(n_{i m m}-\left(n_{i m m}^{2}-\mathrm{NA}_{\mathrm{o}}^{2}\right)^{1 / 2}\right) \approx 250 \mathrm{~nm}$, where $n_{\text {imm }}=1.518$ is the refractive index of the objective immersion oil (Olympus Type F). The optical refocusing module (ETL, Optotune EL-10-30-C) and the sCMOS camera (Hamamatsu, Orca Flash 4.0, 16-bit gray-level, pixel size of $6.5 \mu \mathrm{m}$ ) have been synchronized allowing an automatic axial scanning of 50 refocused images $(400 \times 400$ pixels) in a time of $100 \mathrm{~ms}$. In Fig. 5 (a) two intensity images of the buccal mucosa cell are shown. The RI reconstruction has been performed following the procedure described in Ref. $^{7}$ and it has been done for the part of the cell within the dash line yellow box. Let us consider the RI contrast defined as $\Delta n=n_{s}-n_{m}$ instead of RI itself. In Fig. 5 (b,c) the $x-y$ slices of the $\Delta n$ distribution of the epithelium and the cell itself, correspondingly, are shown. The maze-like structure of the epithelial layer with approximately $300 \mathrm{~nm}$ diameter fibers is clearly observed. The average $\Delta n$ of this maze-like fiber structure is 0.01 , that is above the surrounding medium RI but below the averaged $\Delta n$ value of bacteria (around 0.02) as displayed in Fig. 5 (d). We recall that no post-deconvolution processing has been applied for 
(a) Raw intensity images from buccal mucosa squamous cell $\mathrm{z}=2 \mu \mathrm{m}$

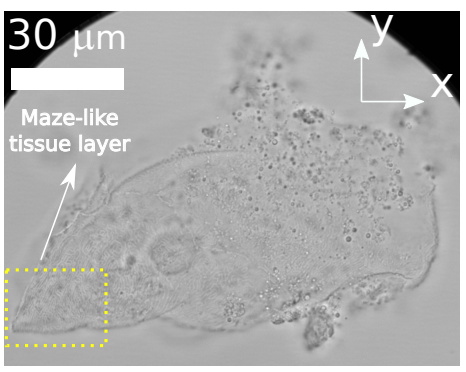

(b) Reconstructed $\Delta n$ in an epithelial layer

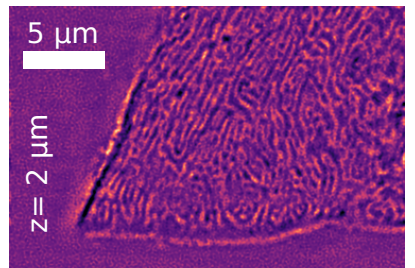

(d) Different points of view from the reconstructed 3D $\Delta \mathrm{n}$

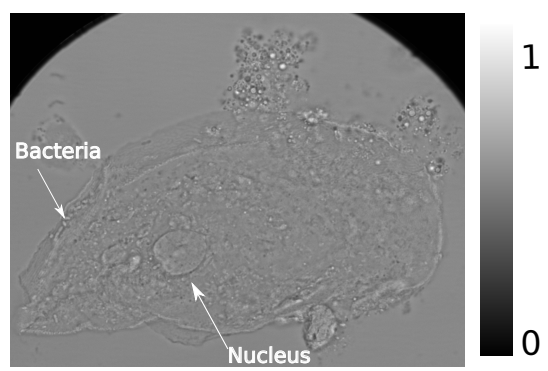

(c) Reconstructed $\Delta \mathrm{n}$ in buccal bacteria layer 0.02

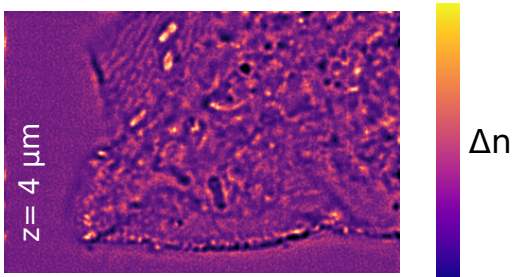

0
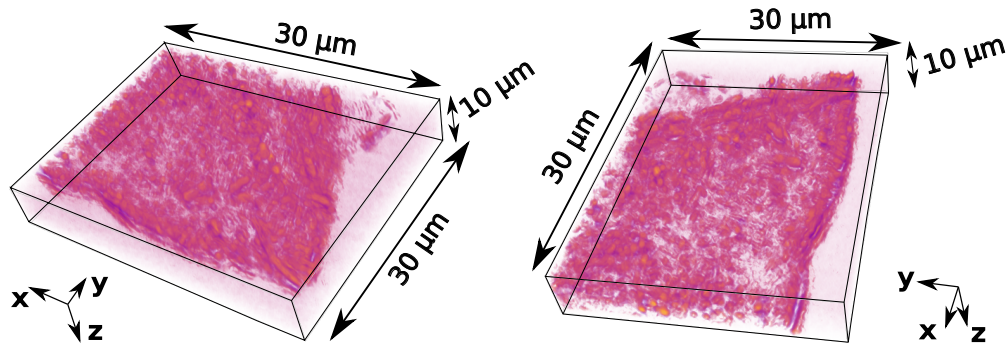

Figure 5. (a) Intensity distributions of the cell image recoded at two different planes, showing the big nucleus of this cheek cell and the presence of bacteria. (b) Reconstructed $\Delta n$ in a epithelial tissue layer, exhibiting a fine maze-like structure of fibers. (c) Reconstructed $\Delta n$ in buccal mucosa cell layers where one can easily detect several bacteria. (d) $3 \mathrm{D}$ visualization of the reconstructed $\Delta n$ renderized with Tomviz imaging tool.

these reconstructions. The 3D $\Delta n$ visualization included in Fig. 5 (d) allows clearly observing the bacteria forms. Note that in Fig. 5 (d) the background has been substracted to make the structure semi-transparent.

\section{CONCLUSIONS}

We have reviewed the advantages and disadvantages of the PC-ODT technique developed for fast label-free high-resolution 3D quantitative imaging compatible with conventional wide-field transmission microscope. By analyzing different type of illuminations applied for PC-ODT we have concluded that the Gaussian illumination is the best choice for this technique implementation. Further work has to be developed in terms of a more careful post-deconvolution processing that takes into account the bias existing in the reconstructed RI in order to better mitigate the missing cone problem.

\section{ACKNOWLEDGMENTS}

The funding of the projects TEC2014-57394-P (Ministerio de Economa y Competitividad) and PR75/18- 21567 (Santander-UCM) is acknowledged. 


\section{REFERENCES}

[1] E. Wolf, "Three-dimensional structure determination of semi-transparent objects from holographic data," Opt. Commun. 1, 153-156 (1969).

[2] Y. Cotte, F. M. Toy, C. Arfire, S. S. Kou, D. Boss, I. Bergoënd, and C. Depeursinge, "Realistic 3D coherent transfer function inverse filtering of complex fields." Biomed. Opt. Express 2, 2216-30 (2011).

[3] Y. Sung, W. Choi, C. Fang-Yen, K. Badizadegan, R. R. Dasari, and M. S. Feld, "Optical diffraction tomography for high resolution live cell imaging," Opt. Express 17, 266-277 (2009).

[4] Y. Cotte, F. Toy, P. Jourdain, N. Pavillon, D. Boss, P. Magistretti, P. Marquet, and C. Depeursinge, "Marker-free phase nanoscopy," Nat Phot. 7, 113-117 (2013).

[5] J. Lim, K. Lee, K. H. Jin, S. Shin, S. Lee, Y. Park, and J. C. Ye, "Comparative study of iterative reconstruction algorithms for missing cone problems in optical diffraction tomography." Opt. Express 23, 16933-48 (2015).

[6] K. Lee, K. Kim, G. Kim, S. Shin, and Y. Park, "Time-multiplexed structured illumination using a dmd for optical diffraction tomography," Opt. Lett. 42, 999-1002 (2017).

[7] J. M. Soto, J. A. Rodrigo, and T. Alieva, "Label-free quantitative 3D tomographic imaging for partially coherent light microscopy," Opt. Express 25, 15699 (2017).

[8] J. A. Rodrigo, J. M. Soto, and T. Alieva, "Fast label-free microscopy technique for 3d dynamic quantitative imaging of living cells," Biomed. Opt. Express 8, 5507-5517 (2017).

[9] J. M. Soto, J. A. Rodrigo, and T. Alieva, "Partially coherent illumination engineering for enhanced refractive index tomography," Opt. Lett. 43, 4699-4702 (2018).

[10] N. Streibl, "Three-dimensional imaging by a microscope," JOSA A 2, 121-127 (1985).

[11] Y. Sung and C. J. R. Sheppard, "Three-dimensional imaging by partially coherent light under nonparaxial condition." J. Opt. Soc. Am. A. Opt. Image Sci. Vis. 28, 554-9 (2011).

[12] M. Chen, L. Tian, and L. Waller, "3D differential phase contrast microscopy," Biomed. Opt. Express 7, 3940-3950 (2016).

[13] Y. Bao and T. K. Gaylord, "Quantitative phase imaging method based on an analytical nonparaxial partially coherent phase optical transfer function," J. Opt. Soc. Am. A 33, 2125 (2016).

[14] M. H. Jenkins and T. K. Gaylord, "Three-dimensional quantitative phase imaging via tomographic deconvolution phase microscopy," Appl. Opt. 54, 9213 (2015).

[15] https://www.tomocube.com/product/ht-microscope/.

[16] https://nanolive.ch/hardware/.

[17] J. Li, Q. Chen, J. Sun, J. Zhang, X. Pan, and C. Zuo, "Optimal illumination pattern for transport-ofintensity quantitative phase microscopy," Opt. Express 26, 27599 (2018).

[18] J. Li, Q. Chen, J. Sun, J. Zhang, J. Ding, and C. Zuo, "Three-dimensional tomographic microscopy technique with multi-frequency combination with partially coherent illuminations," Biomed. Opt. Express 9, 2526$2542(2018)$.

[19] J. Lim, A. Wahab, G. Park, K. Lee, Y. Park, and J. C. Ye, "Beyond Born-Rytov limit for super-resolution optical diffraction tomography," Optics Express 25, 30445-30458 (2017).

[20] K. C. Tam and V. Perezmendez, "Tomographical imaging with limited-angle input," J. Opt. Soc. Am. 71, 582-592 (1981).

[21] B. P. Medoff, W. R. Brody, M. Nassi, and A. Macovski, "Iterative convolution backprojection algorithms for image-reconstruction from limited data," J. Opt. Soc. Am. 73, 1493-1500 (1983).

[22] A. Sakellariou, C. H. Arns, A. P. Sheppard, R. M. Sok, H. Averdunk, A. Limaye et al. "Developing a virtual materials laboratory," Materials Today, 10(12), 44-51 (2007).

[23] V. Lauer, "New approach to optical diffraction tomography yielding a vector equation of diffraction tomography and a novel tomographic microscope," Journal of Microscopy, 205(2), 165-176 (2002).

[24] J. Jung, K. Kim, J. Yoon, and Y. Park, "Hyperspectral optical diffraction tomography," Opt. Express, 24, 2006-2012 (2016). 